\title{
Caracterização histoquímica e ultra-estrutural do estipe de Socratea exorrhiza (Mart.) H. Wendl. (Arecaceae) ${ }^{1}$ \\ Histochemical and ultrastructure characterization of stem of Socratea exorrhiza (Mart.) H. Wendl. (Arecaceae) $)^{1}$
}

Resumo: $O$ objetivo deste trabalho foi caracterizar as substâncias ergásticas presentes no estipe da palmeira Socratea exorrhiza (Mart.) $H$. Wendl., do ponto de vista da microscopia eletrônica de varredura. A análise histoquímica foi realizada com material botânico in natura coletado na ilha do Cumbu, Pará. O reagente lugol foi empregado para constatar a presença de grãos de amido, cristais de fenol para identificar sílica e a solução de ácido clorídrico 10\% + ácido acético glacial para evidenciar cristais de oxalato de cálcio. Os testes revelaram a presença de grãos de amido, cristais de oxalato de cálcio e sílica, porém, suas formas e tipos estruturais só foram discernidos quando analisados em microscopia eletrônica de varredura.

Palavras-chave: Arecaceae. Socratea. Histoquímica. Ultra-estrutura. Amido.

\begin{abstract}
The aim of this work was to caracterize ergastics substances in stem of the Socratea exorrhiza (Mart.) H. Wendl., according to the point of view of the scanning electron microscopy. The histochemical analyses were carried with in natura samples from in the island of the Cumbu, Pará. The lugol was used to evidence the presence of starch grains; phenol crystals to silica; and acid clorídrico 10\% + acid ascetic glacial, to crystals of calcium's oxalate. The scanning electron microscopy showed triangular, ellipse and ellipsoidal starch grains, over there of calcium's oxalate and silica crystals.
\end{abstract}

Keywords: Arecaceae. Socratea. Histochemic. Ultrastructure. Starch.

Parte da Dissertação de Mestrado em Botânica Tropical da primeira autora.

Engenheira Agrônoma (typinheiro@yahoo.com.br).

II Museu Paraense Emílio Goeldi. Coordenação de Botânica. Belém, Pará, Brasil (raipoty@museu-goeldi.br).

III Museu Paraense Emílio Goeldi. Coordenação de Botânica. Belém, Pará, Brasil (santos_pp@yahoo.com.br).

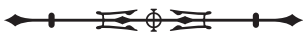




\section{INTRODUÇÃO}

As espécies da família Arecaceae, plantas conhecidas como palmeiras, apresentam grande importância econômica e são exploradas comercialmente na produção de óleo, amido, palmito, cera e fibras (Santelli et al., 2006).

A palmeira Socratea exorrhiza (Mart.) H. Wendl., conhecida popularmente como 'paxiúba' ou 'árvore das pancadas' (Vilhena et al., 1984), encontra-se dispersa por toda Amazônia, habitando terrenos alagados, mal drenados e, algumas vezes, em terra firme; destaca-se por possuir potencial econômico regional, visto que suas partes vegetativas são altamente fibrosas; assim como muitas Arecaceae, o órgão mais utilizado de $S$. exorrhiza também é o estipe, o qual é empregado na construção de paredes e forros de casas (Henderson et al., 1995), porém, atualmente já se conhecem outros empregos, como a confecção de móveis e biojóias.

Estudos etnobotânicos relatam que as partes vegetativas das palmeiras mais utilizadas pelas comunidades amazônicas realmente são os estipes e as folhas, pois apresentam boa resistência mecânica (Ribeiro et al., 1999). Embora estudados anatomicamente, pouco se conhece sobre os metabólicos sintetizados nestes órgãos, uma vez que apresentam uma enorme possibilidade de variação estrutural devido às diferentes orientações e ligações entre moléculas.

Segundo Dietrich (2006), o estudo do conteúdo e composição de polissacarídeos de plantas superiores nativas foi iniciado em órgãos subterrâneos e sementes de leguminosa, identificando grande diversidade de tipos e estruturas, além de uma estreita relação entre os grupos taxonômicos nos quais os diferentes tipos de substâncias são encontrados. De acordo com Menezes Neto et al. (1997), as células vegetais podem produzir diversos tipos de substâncias que podem ser de grande valor taxonômico e filogenético em vários grupos de plantas (Essig, 1999), nos quais, com auxilio da microscopia eletrônica de varredura, se tornou possível ampliar o conhecimento sobre as células vegetais com riqueza de detalhes, juntamente com suas estruturas (Grimstone, 1980).
Assim, o presente trabalho tem como objetivo identificar as possíveis substâncias ergásticas presentes no estipe, enfatizando suas formas e tipos estruturais do ponto de vista da microscopia eletrônica de varredura, como contribuição a futuros trabalhos que visem ao melhor conhecimento fisiológico e bioquímico, além do potencial de utilização das Arecaceae.

\section{MATERIAL E MÉTODOS}

O estipe foi extraído de três espécimes férteis, com 16 m de altura e $18 \mathrm{~cm}$ de diâmetro, coletado em uma área de várzea baixa da ilha do Cumbu, situada na margem esquerda do rio Guamá, com aproximadamente $24,28 \mathrm{~km}^{2}$ de extensão de longitude W 48 29' 34.44"' $48^{\circ} 24^{\prime}$ ' 18.27" Grennwich e latitude S $01^{\circ} 28^{\prime} 14.88^{\prime \prime} / 01^{\circ} 31^{\prime} 13.27^{\prime \prime}$.

Para os testes histoquímicos, foram utilizadas amostras do material, ainda fresco, retirado da região periférica e medular. Destes, foram obtidas seções a mão livre e analisadas ao natural. O lugol (Johansen, 1940) foi empregado para detectar a presença de amido; o ácido clorídrico 10\% (Chamberlain, 1932) para verificar a presença de cristais de oxalato de cálcio; e cristais de fenol (Jensen, 1962) para evidenciar a presença de sílica.

Para o estudo ultra-estrutural, as amostras foram desidratadas em série etílica, levadas à secagem em ponto crítico, montadas em 'Stubs', metalizadas em ouro (Silveira, 1989) e examinadas no microscópio eletrônico de varredura (MEV) LEO 1450 VP, que está acoplado a uma sonda eletrônica capaz de detectar vários elementos minerais, alocado na Coordenação de Ciências da Terra e Ecologia do Museu Paraense Emílio Goeldi (MPEG). As observações da forma e tamanho dos grãos de amido foram determinadas pela escala de medida da fotografia eletrônica de varredura.

\section{RESULTADOS E DISCUSSÃO}

A região periférica do estipe é constituída por fibras perivasculares, de paredes secundárias espessas, fortemente lignificadas e sobrepostas umas as outras, formando 
camadas. Estas ainda são freqüentemente onduladas e geralmente associadas a células pétreas, além de idioblastos contendo cristais do tipo piramidal (Figuras 1a e 1b) e ráfides (Figuras 1c e 1d). A microanálise realizada pela sonda acoplada ao MEV detectou, respectivamente para ambos os cristais, uma elevada quantidade do elemento cálcio próximo ao oxigênio, sugerindo a existência de um composto de cálcio, possivelmente o oxalato (Figuras $2 \mathrm{a}$ e 2b). Idioblastos contendo corpos silicosos também foram detectados pela microanálise, apresentando alta concentração de sílica (Figura 2c) em relação às outras substâncias, como oxigênio, carbono e cálcio. Estes, por sua vez, em microscópio óptico, são esféricos globosos, com a superfície elevada, não sendo possível discernir o aspecto de sua superfície (Figura 1e); porém, ao observá-los em MEV, notou-se com nitidez a superfície espiculada dos corpos silicosos, que estão dispostos em série por todo o comprimento da célula fibrosa (Figura 1f).

Segundo Cutter (1986), através do uso da microscopia eletrônica de varredura com esse tipo de sistema, é possível identificar a distribuição e localização de sílica e cristais de oxalato de cálcio nos órgãos aéreos de Cannabis sativa L., podendo até mesmo detectá-los em restos de cinzas. Como em outras palmeiras, Tomlinson (1961) descreveu os corpos silicosos como estruturas freqüentes ao longo das fibras de Arecaceae, o qual chamou de 'stegmatas' com formato de um chapéu. No entanto, através da imagem refinada desta estrutura em microscopia eletrônica de varredura, foi possível identificar com clareza a aparência real do idioblasto silicoso, revelando um novo aspecto que não se suspeitava que existisse (Grimstone, 1980): as espículas sobre a base do corpo silicoso. Rocha (2004) e Silva (2006) também as observaram nos 'stegmatas' ao longo das fibras das palmeiras Astrocaryum murumuru var. murumuru Mart., Oenocarpus bacaba Mart, O. minor Mart, O. disticus Mart. e O. mapora H. cast, respectivamente em MEV.

Embora a presença de sílica e idioblastos contendo ráfides e monocristais de oxalato de cálcio sejam comuns em Arecaceae (Tomlinson, 1990) e em outras monocotiledôneas como Bromeliaceae, Zingiberaceae, Orchidaceae, Pandanaceae e Cynclantaceae (Dahlgren; Clinford, 1982), questiona-se muito sobre o papel que desempenham no vegetal, devido ao pouco conhecimento fisiológico dos mesmos. Entretanto, Haberlandt (1925) e Paiva (2003) sugerem que os corpos silicosos e os cristais de oxalato de cálcio, respectivamente, formem-se devido à ausência de um sistema excretor especializado. Já Franceschi e Horner Jr. (1980) citam que a função dos cristais de oxalato de cálcio está relacionada ao equilíbrio iônico da planta e, quando presentes na forma de ráfides, tornam-se menos palatáveis aos animais (Mauseth, 1995). Talvez em S. exorrhiza exerçam as mesmas funções.

Quanto à localização, os cristais podem estar presentes nas células parenquimáticas, raios, fibras ou ainda formando séries cristalíferas (Appezzato-da-Glória; Carmello-Guerreiro, 2003), como observado nas células parenquimáticas de $S$. exorrhiza na forma de rafídeos formando cadeias com até 12 células. Vilhena et al. (1984) também observaram a presença desse elemento mineral nas raízes de Iriartea exorrhiza Mart.

Com relação aos cristais de sílica, estes podem ser encontrados na forma de partículas, grãos, agregados amorfos ou ainda como estrutura vítrea (Appezzatoda-Glória; Carmello-Guerreiro, 2003). Esta última foi observada principalmente incrustada na parede das fibras do estipe, provavelmente com função de resistência mecânica (Hanberlandt, 1925). Segundo Windisch (1990), a ação abrasiva existente nos caules de pteridófitas do gênero Equisetum decorre da presença da enorme quantidade de cristais de sílica, que em algumas regiões chega a ser utilizado como substituto da palha de aço na lavagem de panelas. Oliveira e Sajo (2001) sugeriram que a presença dos corpos cônicos de sílica incrustados aos feixes vasculares em nove espécies de Orchidaceae esteja relacionado ao xerofitismo das espécies.

A região medular do estipe em microscopia óptica apresentou abundantes idioblastos repletos de grãos de 

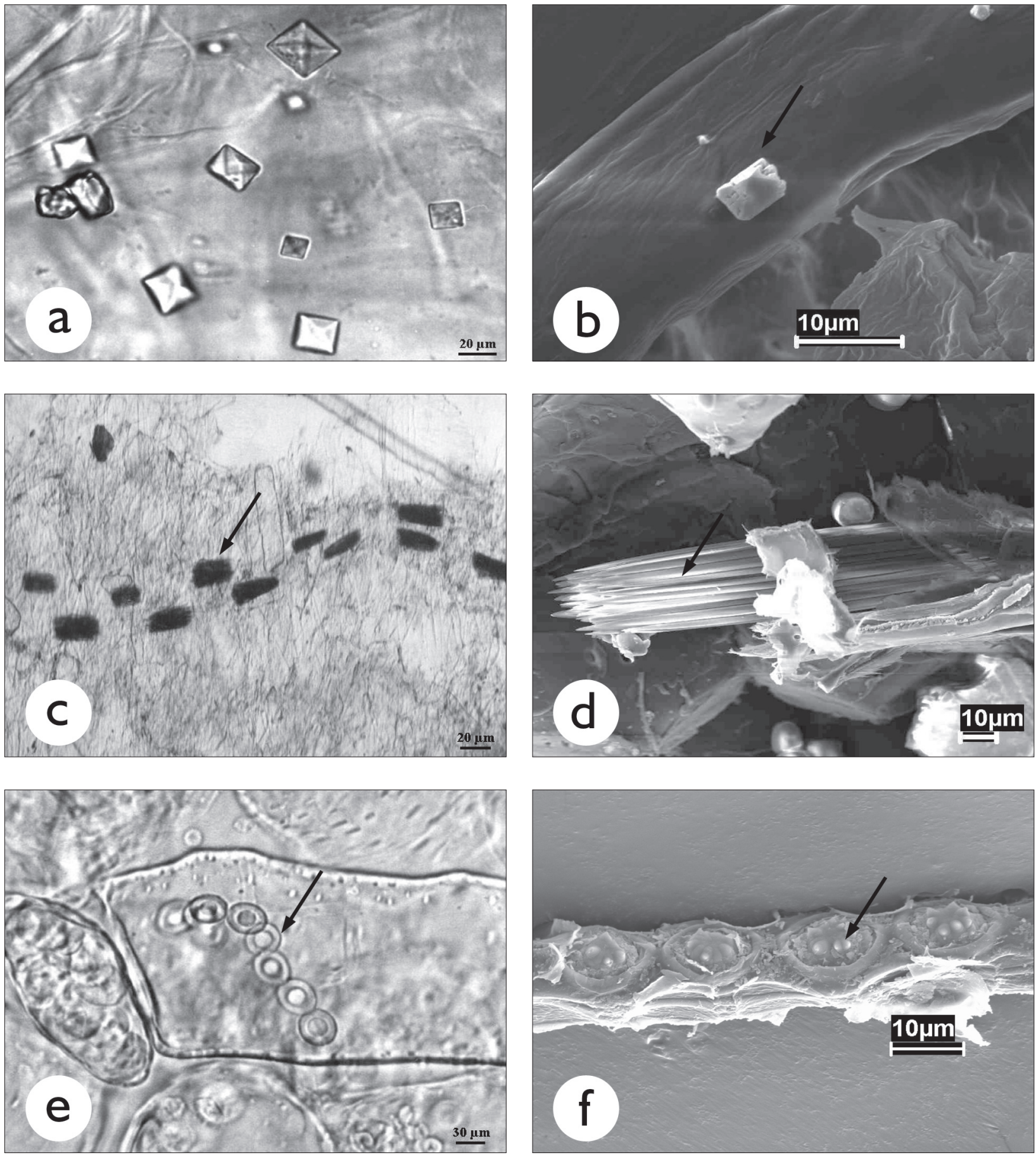

Figura 1. Socratea exorrhiza (Mart.) H. Wendl. Substâncias ergásticas: a) cristais prismáticos em microscopia óptica; b) detalhe do cristal prismático em MEV; c) idioblastos de cristais de ráfides, em séries; d) detalhe do idioblasto de ráfides; e) corpos silicosos em Microscopia Ótica; f) detalhe dos corpos silicosos com várias espículas em MEV, ao longo da fibra. 
amido, que se concentram principalmente próximo aos feixes vasculares. Os grãos de amido apresentam-se simples, de formato predominantemente arredondado, tamanho uniforme e com fissuras ao redor do hilo (Figura 3a). Entretanto, ao observá-los em microscopia eletrônica de varredura, notou-se que os mesmos apresentam uma diversidade de formas (Figura 3b), com grãos semi-compostos e compostos (Figura 3c), estruturas variadas do tipo triangular, elíptico e oval, superfície lisa, encaixados ou livres e ausência de estrias ao redor do hilo (Figura 3d), tamanhos variando de 4,16 a 17,37 $\mu \mathrm{m}$ de comprimento e 3,29 a 12,56 $\mu \mathrm{m}$ de largura. Características estas que só puderam ser evidenciadas com a análise em microscópio eletrônico de varredura.

O potencial amilífero da região medular do estipe de palmeiras foi relatado por Hill (1952), Whistler e Paschall (1967) e Cereda e Vilpoux (2007), que abordaram a extração dos grânulos do amido 'sagu', retirado de Metroxylon sagu Robbt., para fabricação de farinha e tapioca utilizada como complemento alimentar no sudeste asiático.

Segundo Giacometto e Wosicacki (1985), a concentração de polissacarídeos na região medular do estipe deve-se à formação do amido em organelas especializadas, chamadas amiloplastos, as quais, após a formação, precipitam e são dispersas pelo citoplasma celular, localizando-se principalmente ao redor do floema, vaso que transporta sacarose para todas as partes da planta (Daiuto, 2002).

Quanto à diversidade de formas e tipos estruturais observados em microscopia eletrônica de varredura, Esau (1972) e Galliard (1987) relataram que a estrutura do amido varia muito entre espécies, pois cada uma possui certo padrão morfológico de deposição, o que pode interferir na forma e tamanho do grão; este fato é utilizado para identificar espécies quando há dúvidas com relação à sua morfologia externa ou até mesmo comparar espécies atuais com materiais arqueológicos, a fim de verificar se houve evolução da estrutura deste amido ao longo do tempo (Freitas, 2002). Segundo Cutter (1986), os grãos de amido localizados nos latićf́eros de Euphorbia milii Des Moul. são alongados ou em forma de alteres, ao passo que aqueles que constituem as células parenquimáticas adjacentes são ovais.

Outras considerações a respeito do formato dos grãos de amido também foram abordadas por Salisbury e Ross (1992), que descreveram o amido como um polissacarídeo de reserva resultante de dois tipos de polímeros de glucose: um essencialmente linear, chamado
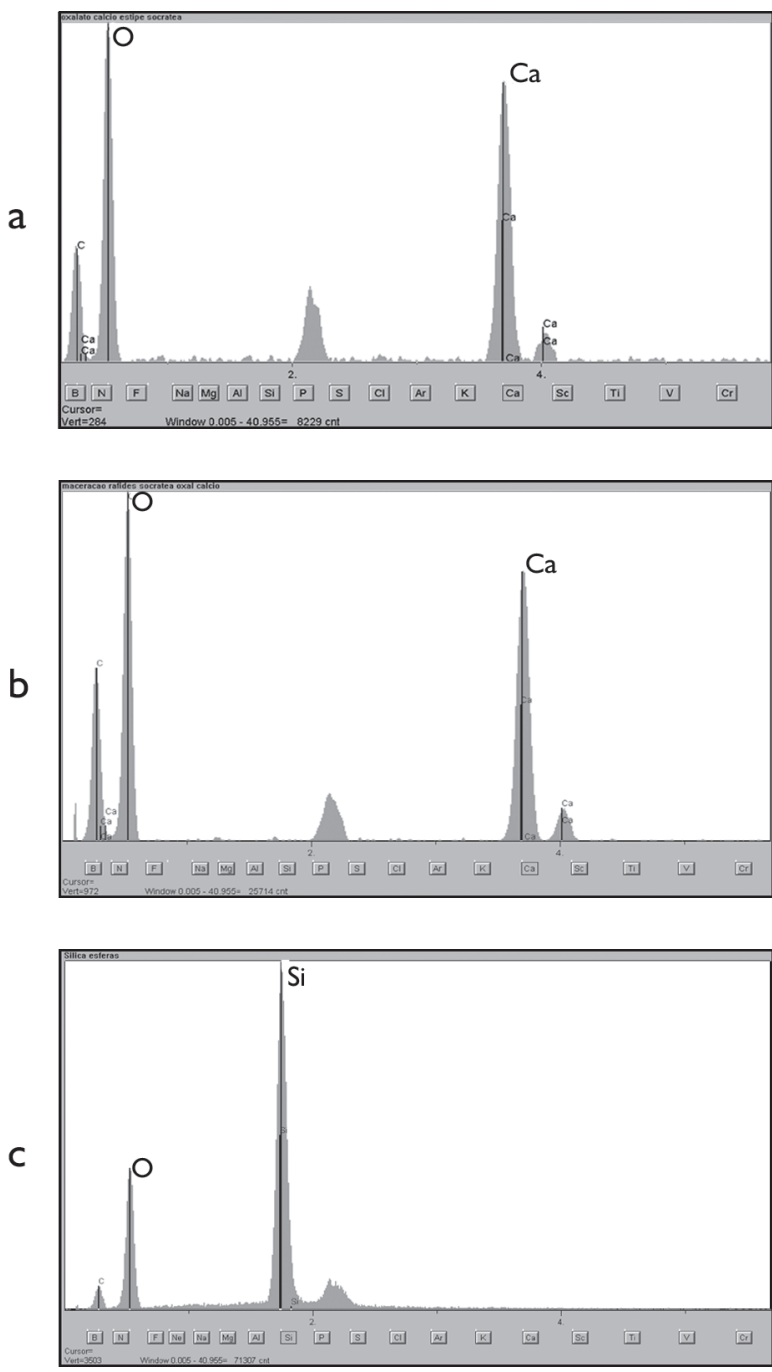

Figura 2. Socratea exorrhiza (Mart.) H. Wendl. Microanálise em MEV: a) presença de oxalato de cálcio nos cristais piramidais; b) presença de oxalato de cálcio nas ráfides; c) presença de sílica nos corpos silicosos. 

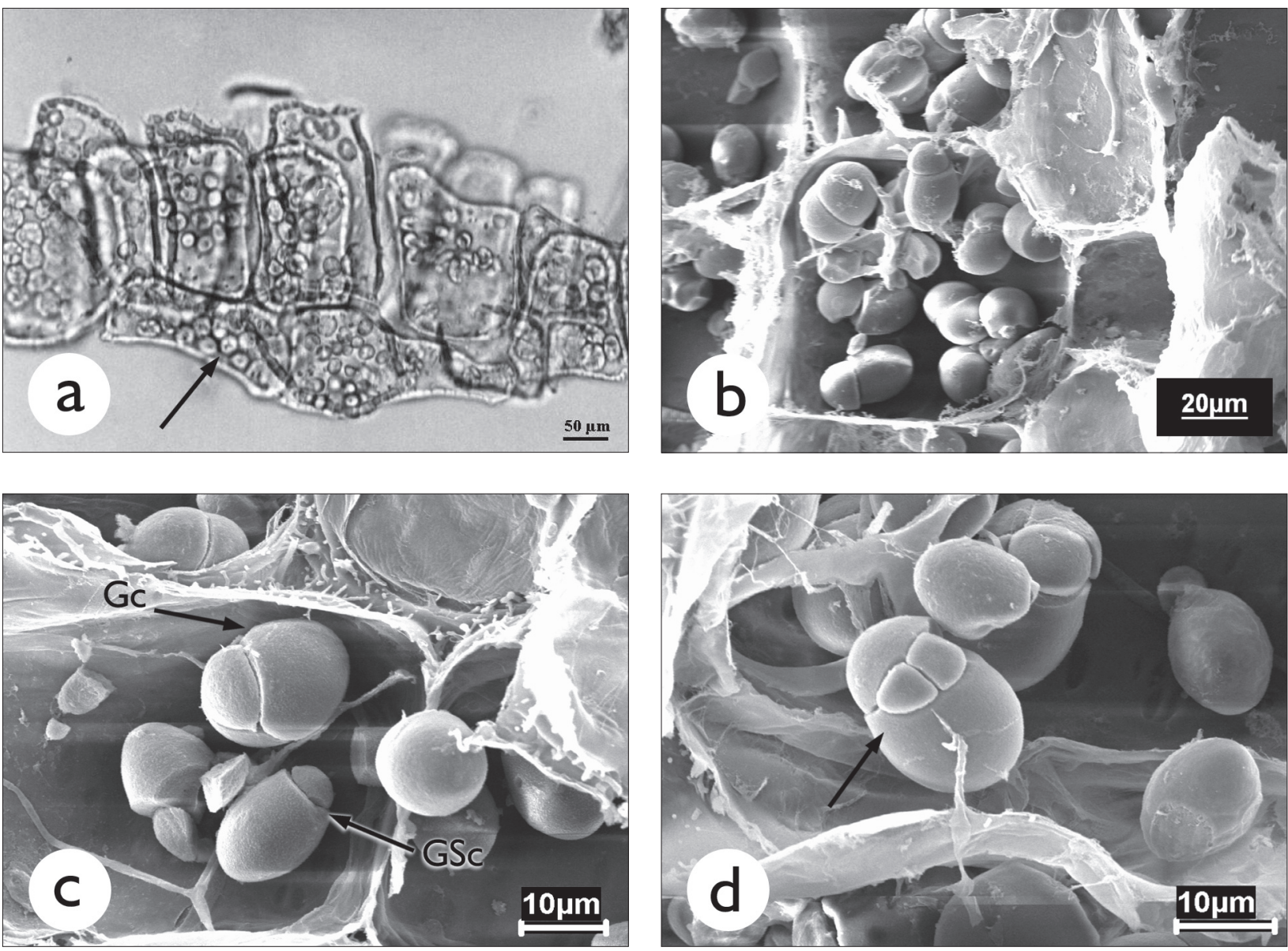

Figura 3. Socratea exorrhiza (Mart.) H. Wendl. Substâncias ergásticas: a) idioblastos amilíferos em microscopia óptica, mostrando fissuras; b) idioblasto em microscopia eletrônica de varredura; c) detalhe dos grãos de amido semi-compostos e compostos; d) detalhe do grão composto, ovalado, encaixado, sem estrias na superfície. Gc - grão composto; GSc - grão semi-composto.

de amilose e outro altamente ramificado, chamado amilopctina, cujo formato pode variar desde formas mais esféricas até elípticas, o que é determinado em grande parte pelo conteúdo de amilose, pois o grão de amido torna-se mais esférico na medida em que ocorre aumento do conteúdo da amilose em relação ao de amilopectina (Bewley; Black, 1994). Assim, provavelmente, os grãos observados nas células parênquimaticas do estipe de S. exorrhiza apresentem quantidades maiores de amilopectina e, por esta razão, adquirem o formato elíptico ou ovalado neste estágio de desenvolvimento, uma vez que, em geral, os grânulos de amido têm sua estrutura e propriedades funcionais alteradas durante o desenvolvimento da planta (Cereda; Franco, 2002). Amaral et al. (2001) relataram para as sementes de Bixa orellana L. maior depósito de amilopctina apenas nos estágios iniciais de desenvolvimento e em fases mais avançadas de amilose, adquirindo formato esférico.

Outra característica relevante foi quanto ao tamanho dos grãos. Estes podem variar com a idade e com o próprio desenvolvimento do órgão de reserva (Daiuto, 2002); assim, grãos de uma mesma planta podem apresentar diâmetros diferentes (Sarmento, 1997). Baseados nesta característica, Ugent e Verdum (1983) confeccionaram uma chave dicotômica para facilitar o reconhecimento e separação de espécies do

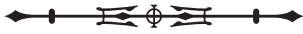


gênero Solanum. Leonel et al. (2002), analisando os grãos de amido de Canna edulis Ker Gawl., observaram formatos ovalados, circulares e achatados, que variavam entre 9 a $65 \mu \mathrm{m}$ de comprimento e 4 a $50 \mu \mathrm{m}$ de largura; intervalos estes que enquadram as médias observadas em S. exorrhiza, que apresentou formato dos grãos semelhante aos descritos para mandioca (Nougarède, 1969), podendo-se sugerir um melhor aprofundamento de suas características físico-químicas para melhor compreensão dos aspectos de sua funcionalidade e conseqüente determinação de uso pela indústria.

\section{CONCLUSÃO}

A microscopia eletrônica de varredura permitiu a identificação das formas e tipos estruturais das substâncias ergásticas presentes no estipe de $S$. exorrhiza, sendo mais refinado que o óptico e de grande relevância para estudos minuciosos do metabolismo das mesmas. As substâncias ergásticas encontradas no estipe foram grãos de amido de formato semi-composto e composto, do tipo triangular, elíptico e oval, sem a presença de estrias ao redor do hilo; cristais de oxalato de cálcio em forma de ráfides e prismáticos; além de corpos silicosos espiculados.

\section{AGRADECIMENTOS}

Agradecemos ao Dr. Hilton Túlio Tosti, pela dedicação na obtenção das imagens em (MEV); e à CAPES, pela concessão de bolsa à primeira autora.

\section{REFERÊNCIAS}

AMARAL, L. I. V. do; PEREIRA, M. de F. D. A.; CORTELAZZO, A. L. 2001. Formação das substâncias de reserva durante o desenvolvimento de sementes de urucum (Bixa orellana L. - Bixaceae). Acta Botanica Brasilica, v. 15, n. 1, p. 125-132, 2001.

APPEZZATO-DA-GLÓRIA; CARMELLO-GUERREIRO, S. M. Anatomia Vegetal. Viçosa: UFV, 2003. p. 136-137.

BEWLEY, J. D.; BLACK, M. Seeds: physiology of development and germination. 2. ed. New York: Plenum Press, 1994. 445 p.

CEREDA, M. P.; FRANCO, C. L. Propriedades gerais do amido. São Paulo: Fundação Cargill, 2002. 221 p., v. 1.
CEREDA, M. P. ; VILPOUX, O. Processos de fabricação de sagu, tapioca e farinha de tapioca. Disponível em: <http://www.mal. cefetpr.br/intranet/professores/adm/download/apostilas/171959. pdf. > Acesso em: 18 Abr. 2007.

CHAMBERLAIN, C. J. Methods in plant histology. 5. ed. Chicago: University of Chicago Press, 1932. 416 p.

CUTTER, E. G. Anatomia vegetal: Parte I - Células e tecidos. Botucatu: Roca, 1986. 304 p.

DAHLGREN, R. M. T.; CLIFFORD, H. T. The monocotyledons: a comparative study. London: Academic Press, 1982. p. 38-103.

DAIUTO, E. R. Desenvolvimento de grãos de amido durante o crescimento secundário de raízes de mandioca dos cultivares Mico e Branca de Santa Catarina. Botucatu, 140 p. Dissertação (Mestrado em Agronomia/Horticultura), Faculdade de Ciências Agronômicas. Universidade Estadual Paulista, 2002.

DIETRICH, S. M. C. A biodiversidade sob a ótica da bioquímica de polissacarídeos de plantas. In: IX CONGRESSO LATINOAMERICANO DE BOTÂNICA, 2006. São Domingos. Libro de Resúmenes. (Conferencia Magistrales). Santo Domingo, 2006, p. 10.

ESAU, K. Anatomia vegetal. 2. ed. Barcelona: Omega, 1972. p. 226-232.

ESSIG, F. 1999. Trends of specialization in the palm pericarp. In: HENDERSON, A. BORCHSENIUS, F. Evolution, Variation, and classification of palms. New York: Botanical Garden, 83: 73-77.

FRANCESCHI, V. R.; HORNER Jr., H. T. Calcium oxalate crystals in plants. The Botanical Review, v. 46, n. 4, p. 361-427, 1980.

FREITAS, F. de O. Uso de grãos de amido na identificação e análise de materiais arqueológicos vegetais. Brasília: Embrapa Recursos Genéticos e Biotecnologia. Boletim de Pesquisa e Desenvolvimento, n. 23, 2002. 26 p.

GALLIARD, T. Starch availability end utilization. In GALLIARD, T. Starch: properties and potential. Brisbane: Jonh Wiley and Son 1987. 1-15 p.

GIACOMETTO, A. P.; WOSIACKI, G. Grânulos e pastas de amido: o estado da arte. Semina, v. 6, n. 3, p. 155-159, 1985.

GRIMSTONE, A. V. O Microscópio eletrônico em biologia. São Paulo: EPU, São Paulo, 1980. 70 p.

HABERLANDT, G. Physiological plant anatomy. Delhi: Today \& Tomorrow`s, 1925. p. 529-613.

HENDERSON, A. GALEANO, G.; BERNAL, R. Field guide to the palms of the Américas. Princeton: Princeton University Press, 1995. $352 \mathrm{p}$.

HILL, A. F. Economic Botany: a textbook of useful plants and plant products. New York: McGraw-Hill Book, 1952. p. 9-12. 
JENSEN, W. A. Botanical histochemistry: principle and pratice. San Francisco: W. H. Freeman, 1962. 408 p.

JOHANSEN, D. A. Plant microtechnique. New Deldi: MacGrawHill, 1940. 523 p.

LEONEL, M.; SARMENTO, S. B. S.; CEREDA, M. P.; GUERREIRO, L. M. R. Extração e caracterização do amido de Biri (Canna edulis). Braz. J. Food Technol., v. 5, p. 27-32, 2002.

MAUSETH, J. D. Botany an introduction plant biology. 2. ed. Menlo Park: Sanders College Blishing, 1995. 560 p.

MENEZES NETO, M. A.; MENDES, A. M. C. de M.; MENDES, A. C. de B. Práticas de anatomia vegetal. Belém, 1997. p. 15 - 25

NOUGARÈDE, A. Biologie végetale. I - Cytologie. Masson, Paris: [s.n.], 1969. p. 310-349.

OLIVEIRA, V. del. C; SAJO, Maria das Graças. Morfo-anatomia caulinar de nove espécies de Orchidaceae. Acta Botanica Brasílica v. 15, n. 2, p. 177-188, 2001.

PAIVA, E. A. S. Possíveis papéis dos cristais de oxalato de cálcio em vegetais. In: Desafios da botânica brasileira no novo milênio: inventário, sistematização e conservação da diversidade vegetal. Belém: MPEG/ UFRA/EMBRAPA, 2003. p. 299-301.

RIBEIRO, J. E. L. da S.; HOPKINS, M. J. G.; VICENTINI, A.; SOTHERS, C. A.; COSTA, M. A. da S.; BRITO, J. M. de; SOUZA, M. A. D. de; MARTINS, L. H. P.; LOHMANN, L. G. ASSUNÇÃO, P. A. C. L.; PEREIRA, E. da C.; SILVA, C. F.; MESQUITA, M. R.; PROCÓPIO, L. C. Flora da Reserva Ducke: guia de identificação das plantas vasculares de uma floresta de terra-firme na Amazônia Central. Manaus: INPA, 1999. p. 655

ROCHA, C. B. R. Anatomia das folhas e análise quantitativas das fibras de Astrocaryum murumuru var. murumuru Mart. (Arecaceae). 57 p. Dissertação (Mestrado em Botânica Tropical) - Museu Paraense Emílio Goeldi, Belém, 2004.

SALISBURY, F. B.; ROSS, C. W. Plant physiology. 4. ed. Belmont: Wadsworth, 1992. p. 267.
SANTELLI, P.; CALBO, M. E. R.; CALBO, A. G. Fisiologia pós-colheita de frutos da palmeira Syagrus oleraceae (Mart.) Becc. (Arecaceae). Acta Botanica Brasilica, v. 20, n. 3, p. 523-528, 2006.

SARMENTO, S. B. S. Caracterização da fécula de mandioca (Manihot esculenta C.) no período de colheita de cultivares de uso industrial. 162 p. Tese (Doutorado em Farmácia). Faculdade de Ciências Farmacêuticas, Universidade de São Paulo, 1997.

SILVA, R. J. F. da. Anatomia foliar comparada em espécies de Oenocarpus Mart. (Arecaceae) de Belém, Pará, Brasil: uma contribuição taxonômica. 2006, 112 p. Dissertação (Mestrado em Botânica Tropical) (de Mestrado em Botânica Tropical) Belém, Museu Paraense Emílio Goeldi, 2006.

SILVEIRA, M. О. O preparo de amostras biológicas para microscopia de varredura eletrônica de varredura. In: SOUZA, W. de (Ed). Manual sobre técnica básicas em microscopia eletrônica de varredura, Técnicas básicas. Sociedade Brasileira de Microscopia Eletrônica, v. 1, p. 71-82, 1989.

TOMLINSON, P. B. Anatomy of the monocotyledons - II Palmae. Oxford: University. London Press, 1961. 440 p.

TOMLINSON, P. B. The strutural biology of palms. Oxford: Claredon Press, 1990. $460 \mathrm{p}$

UGENT, D.; VERDUN, M. Starch grains of the wild and cultivated Mexican species of Solanum, subsection Potatoe. Phytologia, v. 53, p. 351-362, 1983

VILHENA, R. C. Q.; LINS, A.; LUNA, M. S. Estudo anatômico, morfológico e o crescimento das raízes adventícias de Iriartea exorrhiza Mart. (Palmae), "Paxiúba". Bol. Mus. Para. Emílio Goeldi, ser. Botânica., v. 1, n. 1/2, p. 87-109, 1984.

WHISTLER, R. L.; PASCHALL, E. F. Starch: chemistry and tecnology 2 ed. New York: Academic Press, p. 108-119, 1967.

WINDISCH, P. G. Pteridófitas da região Norte-ocidental do Estado de São Paulo: guia para estudo e excursões. São Paulo do Rio Preto, UNESP, 1990. 108 p. il. 